\title{
The Network Process Simulation of Soil-Moisture Infiltration Information System
}

\author{
Hu Hua*
}

School of Mathematics and Computer Science, Ningxia University, Yinchuan City, 750021, China

\begin{abstract}
This paper presents the techniques employed for solutions of the Network Process simulation of Soil-Moisture Infiltration for Soil-Moisture Infiltration Information System. A new approach that uses both enlightened and Random Selection approach is presented to unravel the issues. The usage of random functions comprising of first high value attribute variable and their corresponding combinations are elaborated in this research to overcome some weakness issues utilizing classical public functions. The classification of the Soil-Moisture Infiltration Information System is achieved by using random methods. It has been proved that usage of this new approach based on random selection methods for calculating weights improves precision performance and also minimizes the time required for computations. This research also validates that this approach is concise and more obvious for the decision maker (DM) as compared to the other available techniques.
\end{abstract}

Keywords: Classification, flexibility-function, network, random selection method, soil-moisture infiltration information system.

\section{INTRODUCTION}

The Classification of the object mathematical Mechanism is used for determining its variables relying on experimenal investigation of the object. The main process in the synthesis mechanism is Classification and this process also requires maximum time for its completion.

The classification problem is solved by first determining the relationship between input and output $y=F(x)$ utilizing classical approach. The rules governing this transformation between the input $x$ and output $y$ are applied. The operator $F$ is also investigated for finding its variables and form. This classification approach is called direct because it relies measuring object's output and input signals quantitatively. When output signal information is not available for some situations then the identification of the object is carried out. It is assumed that the objects mentioned in this research are also of this nature.

In some cases, it is not physically possible to measure properties and provide estimated values due to the pertaining nature of the object. The direct classification methods can not be applied for such scenarios and different methods available for indirect classification are employed. The classbased classification method is largely utilized and it is the most common method for such situations [1].

\section{THE RANDOM DEPENDENCE OF SOIL- MOISTURE INFILTRATION}

Assume that a separate set of solutions $X=\left\{x_{j}\right\}$, $j=1, \ldots, m$, is available. Each alternatives is uniquely dependant on the individual criteria $k_{i}, i=1, \ldots, n$ These criteria $k_{i}\left(x_{j}\right)$ values are primarily available. The proper and effective solution from set of solutions $X$, for example $x_{j}$, i.e can be chosen. A dependence relation on set of alternatives $X$ can be written as follows:

$$
x_{1} \geq x_{2} \geq \ldots \geq x_{m}
$$

The preference of any alternative $x_{j} \in X$ considering utility theory is postulated which can be written as follows:

$P\left(x_{i}\right)>P\left(x_{j}\right) ; \forall j \neq i ; j=1, \cdots, m$.

where $\mathrm{P}\left(\mathrm{x}_{\mathrm{j}}\right)$ presents the scalar value of feasibility for alternatives.

The mathematical mechanism of unique decision maker is synthesized by relying on above feasible solutions i.e, a Mechanism of Inherited Attributes utility formation $\mathrm{P}\left(\mathrm{x}_{\mathrm{i}}\right)$.

Nowadays, the additive form of public function is in common use represented as:

$$
P_{1}(x)=\sum_{i=1}^{n} \lambda_{i} k_{i}(x)
$$

and multiplicative form is given as follows:

$$
P_{2}(x)=\prod_{i=1}^{n} \lambda_{i} k_{i}(x)
$$

where $\lambda_{\mathrm{i}}$ denotes the isomorphism coefficients which shows range values, importance, dimensions and criteria $k_{i}$ that portrays the type of isomorphism. 
When isomorphism coefficients are numerically available, the nature of the solution is well known and maximum information is available. $\lambda_{i}$ represents constant, then (3) can be presented as given below:

$$
P_{k}(x)=\prod_{i=1}^{n} \lambda_{i} \prod_{i=1}^{n} k_{i}(x)
$$

The insight of (4) reveals that multiplicative estimation does not consider the partial weighting criteria. The term $\prod_{i=1}^{n} \lambda_{i}$ is a constant scaling multiplication factor and does not contribute to the available solutions $x \in X$. Hence, an additive public function is a natural choice and is considered best optimum and effective function.

If unique criteria and isomorphism coefficients are not provided in $\lambda_{i}$ calculations, then issues arise, so it is assumed to rewrite Equation (2) interms of additive public function as given:

$$
P(x)=\sum_{i=1}^{n} a_{i} k_{i}^{H}(x)
$$

where $a_{i}$ represents the dimensionless coefficients of relative weight that ensures these conditions.

$$
0 \leq a_{i} \leq 1, \quad \sum_{i=1}^{n} a_{i}=1
$$

$k_{i}^{H}(x)$ is normalized, i.e. converted to the partial isomorphic criteria.

The expression used for carrying out normalization process is given as below:

$k_{i}^{H}(x)=\left(\frac{k_{i}(x)-k_{i_{w}}}{k_{i_{B}}-k_{i_{w}}}\right)$

where $k_{i}(x)$ represents values for private criteria; $k_{i_{B}}, k_{i_{w}}$ - represents the desireable and most undesireable values for this criteria that lies in the range of accepted values.

The highest priority coefficients are given more weightage in the parametric classification to overcome issues arising in the synthesis of public functions. For this purpose, the methods employed are based on evaluation or class based methods [3].

The dependency of public function does not rely randomly on absolute values of $k_{i}$ criteria and their respective effects. This is considered a weak point when considering an additive public function.

The effective and optimum solution under less supposed conditions of structure mechanism comes out from the Inher- ited Attribute internal variable Network Process simulation. This solution is theoretically and practically interesting as well.

The polynomial obtained from this structure class procedure is written as follows:

$$
\begin{gathered}
P(x)=a_{0}+\sum_{i=1}^{n} a_{i} k_{i}^{H}(x)+\sum_{r=n+1}^{m} \sum_{g=1}^{n} a_{r} k_{i}^{H}(x) * k_{g}^{H}(x)+\cdots \\
i=1, \cdots, n ; g=1, \cdots, n, r=n+1, \cdots, m .
\end{gathered}
$$

Random Selection methods is applied as a technique for solution of the Inherited Attribute internal variable Network Process simulation of Soil-Moisture Infiltration.

This approach allows us to describe any random dependence. Any limitation arising from multiplicative or additive functions are not taken into account using this approach. The term $k_{i}(x)$ in polynomial (8) is responsible for the features of first and higher degree and all its respective variations.

\section{INFILTRATION OPTIMAL COMPLEXITY MECHANISM}

An optimum solution is the first preference for Process simulation of Soil-Moisture Infiltration which is synthesizable using an Infiltration Optimal Complexity Mechanism. This approach is prone to errors produced due to approximating criterias in experimental output Mechanism.

The algebraic equations solutions are utilized for approximation of $N$ experimental data which represent $N-1$ degree polynomials. This does not ensure synthesis of the approximated data, because many unknown and measured errors arise in this process. This high complex polynomial takes into account of this approximation of required signal and errors produced in data using this technique are also reduced. The data is divided into two sub sets to overcome this issue $[3,4]$. There is also requirement of testing and training. Firstly, the least square method is used for determining characteristics. Secondly, the accuracy and precision is also checked for this approach. If complexity of this approach rises then the precision of approximated data also improves until its value reaches to the lowest. After this lower value, there is rapid fall in this value due to damaging components produced randomly.

The approach which produces minimum error in approximation and test sequence is called Infiltration Optimal Complexity Mechanism [3]. There is need to produce precise evaluation criteria of the mathematical approach. The least square approach is mostly employed for classification. In this mechanism, input is taken as numerical values and experimental values as output is required. If classification of Soil-Moisture Infiltration Information System is considered, the exact numerical output values are not available. Many problems arise in this classification approach due to some conditions which are considered below:

\section{NETWORK PROCESS SIMULATION OF INFIL- TRATION MECHANISM}

In the above formulation, many techniques can be applied for solving the class internal variable Network Process 
simulation of Soil-Moisture Infiltration. The requirement to develop a procedure for the approach is common among all mechanisms.

Inherited Attributes of the structure'sMechanism;

definie the parameters vaue quantitatively;

Make a guess of mechanism's quality.

Various combinations of methods, of different precision, complexity, versatility, for the first and second stages are possible. More research is needed in applying these approaches to evaluate error free and versatile criteria field. Simulations were carried out by randomly selected programming approaches and results were investigated for the specific approach.

Random Selection Methods (RSM) utilize natural selection and "survival of the fittest" approach as a primary method. The evolution is based on search techniques and RSM also shape search methods. The older data set in Inherited Attribute are replaced by new additional sections having effective properties. There is also requirement of producing new sequences artificially [5-9].

RSM is primarily based on solutions termed as population. Each unique element of population is referred as Inherited Attribute and they provide final results for the issues. This process takes multiple runs to calculate Population values and these are found by approximation of flexibilityfunction $[6,7,10]$.

To find solution for internal variable classification, such value of Inherited Attribute is firstly assumed, which clearly depicts the Mechanism's structure. This is established by selecting acceptable structures. Assuming a polynomial excluding some irrelevant terms and restricting certain quadratic and linear parts results in expression as shown below:

Then the polynomial can be represented as follows:

$$
\begin{gathered}
\prod=\sum_{i=1}^{n} a_{i} k_{i}(x)+\sum_{j=1}^{n} a_{j} k_{i}^{2}(x)+\sum_{l=1}^{C_{n}^{2}} a_{l} k_{i}(x) * k_{r}(x) \\
l=1, n ; r=1, n, l \neq r
\end{gathered}
$$

This shows that the resultant polynomial will take the form considering $\mathrm{n}$ partial criteria.

$$
N=2 n+C_{n}^{2}
$$

where $C_{n}^{2}$ represents total combinations available and is expressed as:

$C_{n}^{2}=\frac{n !}{2 !(n-2) !}=\frac{n !}{2(n-2) !}$

Finally, the $N$ bits are available in each Inherited Attributes.

The formula (7) is used for normalization and partial values lie in the $0 \leq k_{i}^{H} \leq 1$. By restricting polynomial complex nature, values residing in a specific region can be obtained. There is rapid decline in values if multiplication or squaring of any two numbers is carried out. A coefficient is multiplied by each polynomial term $a_{i}<1$.

$$
\text { (because } \sum_{i=1}^{N} a_{i}=1 \text { ) }
$$

The terms in the public function which are more than second order are ignored because it is not useful practically to use these high order terms in manipulation of weights $a i$ and public functions $P(X)$.

The parametric classification by utilizing inherited population approach elaborating Mechanism structure in next second phase is calculated by any suitable methods described below:

The Chebyshev point on polyhedron which uses system of inequalities is calculated as follows:

$$
P\left(x_{l}\right)>P\left(x_{j}\right), \quad \forall j \neq l
$$

The Random Selection methods method.

The approaches presented in [2,5] are ignored. For RSM to implement, first $M$ number of coefficients in same amount as $a i$ units are computed. A certain criteria needs to be satisfied for these coefficients:

$$
0 \leq a_{i} \leq 1, \sum_{i=1}^{M} a_{i}=1
$$

The coefficients are written up to two decimal figures. Therefore, the Inherited Attributes of each coefficient is equal to the number of bits as suggested in $[8,10]$ :

$2^{m_{j}-1}<\left(b_{j}-a_{j}\right) * 10^{2}<2^{m_{j}}-1$

where $[a i, b i]$ represents the interval range of $a i$, as depicted in (12), and the coefficient's final value of Inherited Attributes $a i, i=1, \ldots, M$ is:

$B=L * M$

The inherited Attributes coefficients ai are computed and their population is formed, they result in maximizing match function. The Equation (9) representing match function is considered which also satisfies inequalities present in the term. If there is requirement then the test and training set are also devised.

The iteration is used for Random Selection for the first two populations for acquiring the best effective and precise value of the match function.

Example: Suppose five alternatives having four different criteria are available: processor frequency, memory size, hard disk capacity and price. Table $\mathbf{1}$ is made by decision maker for selecting best alternative among all.

The formula (7) is used for finding defined highest and lowest values of criteria and normalized values of partial criteria are also computed from this expression.

The normalized criteria for partial terms are given in Table 2 , which are calculated after carrying out all above procedures. 
Table 1. The alternative's set with partial criteria.

\begin{tabular}{|c|c|c|c|c|}
\hline & K1 & K2 & K3 & 40 \\
\hline$R 1$ & 1100 & 1100 & 80 & 1610 \\
\hline \hline$R 2$ & 610 & 196 & 50 & 2400 \\
\hline$R 3$ & 910 & 260 & 70 & 1200 \\
\hline$R 4$ & 600 & 258 & 60 & 2300 \\
\hline
\end{tabular}

Table 2. Normalized criteria alternative's set.

\begin{tabular}{|c|c|c|c|c|}
\hline & K1 & K2 & K3 & 0 \\
\hline \hline$R 1$ & 1 & 0 & 1 & 0.266 \\
\hline$R 2$ & 0 & 0.531 & 0.24 & 0.732 \\
\hline$R 3$ & 0.76 & 1 & 0.76 & 0 \\
\hline$R 4$ & 0 & 1 & 0.5 & 0.266 \\
\hline
\end{tabular}

The best alternative is selected by DM. If it is supposed that the fourth alternative is chosen by DM, then in the next phase, the additive public function is computed. Firstly, the weight coefficients are obtained and then applied in linear polynomial. The expression for public function is represented as follows:

$P_{a \partial \partial}=0.61 k_{2}+0.39 k_{4}$

The RSM sequence is as follows:

Suppose that the two Inherited Attributes are available: A parent comprising of full information about polynomial and the child that consists of first term elements. The shortest polynomial which is used for obtaining solutions is Child Inherited Attribute and it should satisfy certain conditions given as below:.

$R_{4}>R_{1} R_{4}>R_{2} R_{4}>R_{3} R_{4}>R_{5}$

The Equation (16) is used for selecting the criteria.

The classification based on structural parameters is solved by using above RSM. The criteria in (16) is fulfilled and child Inherited Attributes are obtained.

In the next stage, the optimal length public function as described in polynomial is selected. The shortest polynomial that satisfies criteria in (16) and also have highest value of public function. To develop such polynomial, A condition must also be fulfilled.

$\Delta=P_{\text {gen }}-P_{\text {add }}, \Delta \rightarrow \max$

After this atep is achieved, the next task is to obtain certain lengths for the partial criteria

$P_{\text {gen }}=0.429 k_{2}^{2}+0.571 k_{4}^{2}$
The public functions for different alternatives are as follows: $\mathrm{R} 4=1 ; \mathrm{R} 3=0.429 ; \mathrm{R} 2=0.4277 ; \mathrm{R} 5=0.04 ; \mathrm{R} 1=$ 0.04 .

The maximum value for Public function is R4. Hence, this problem is computed correctly by utilizing this approach.

\section{CONCLUSION}

The random methods utilized for classification of SoilMoisture Infiltration Information System have validated that new approaches like using public functions and random selections methods for obtaining weights results in improvements in time calculation and performance. This approach produces effective results and hence is more preferable as compared to other approaches.

\section{CONFLICT OF INTEREST}

The authors confirm that this article content has no conflict of interest.

\section{ACKNOWLEDGEMENTS}

This study is supported by Natural Science Foundation of Key Projects in NingXia (NZ13008).

\section{REFERENCES}

[1] Ruo hu, Channel Access Controlling in Wireless integrated information Network using Smart Grid System. Applied Mathematics \& Information Sciences. 813-820, 11 (2012)

[2] Ruo hu, Stability Analysis of Wireless integrated information Network Service via Data Stream Methods. Applied Mathematics \& Information Sciences, 793-798, 11 (2012)

[3] G. Ricart and A. Agrawala, An Optimal Algorithm for Mutual Exclusion in Computer Networks. Communications of the ACM. 9$17,24(1981)$ 
[4] M. Maekawa, A sqrt(n) Algorithm for Mutual Exclusion in Decentralized Systems. ACM Transactions on Computer Systems. 145$159,3(1985)$

[5] Hu Ruo, New Network Access Control Method Using Intelligence Agent Technology. Applied Mathematics \& Information Sciences. 44-48, 2 (2013)

[6] Y. Cheng and W. Zhuang, Autonomous Coordination for Fast Handoff in Wireless Mobile Internet. IEEE Communications Magazine. 130-131,5( 2002)
[7] A. Siberschatz and P. B. Galvin, Editor, Operating Systems Concepts, 5th Edition, John Wiley and Sons, Inc., New York, (2008)

[8] B. A. Frouzen, Editor,Data Communications and Networks, Tata McGraw Hill, Noida, (2006)

[9] A. S. Tanenbaum, Editor, Parallel Operating Systems, Prentice Hall, Upper Saddle River, (1995)

[10] K. Hwang, Editor, Advanced Computer Architecture, McGraw-Hill Series in Computer Engineering Inc. Publishing, New York, (1993)

Received: September 16, 2014

Revised: December 23, 2014

Accepted: December 31, 2014

(c) Hu Hua; Licensee Bentham Open.

This is an open access article licensed under the terms of the Creative Commons Attribution Non-Commercial License (http://creativecommons.org/licenses/by-nc/3.0/) which permits unrestricted, non-commercial use, distribution and reproduction in any medium, provided the work is properly cited. 